\title{
Circadian Medicine in Digital Era: New Technologies for New Normal?
}

\author{
Seog Ju Kim \\ Department of Psychiatry, Sungkyunkwan University School of Medicine, Samsung Medical Center, Seoul, Korea
}

The modern person can stay awake at any time of day with the help of electric light. In the 21st century, both the internet and smartphones are commonly used by most people. In the current pandemic era, researchers and clinicians are paying close attention to new technologies, including artificial intelligence, digital therapeutics, and consumer technologies. Just as the use of electricity shifted the human circadian rhythm in the past, these new technologies will be able to provide new strategies for circadian medicine, and expectations for them are high.

Artificial intelligence has led to novel, alternative diagnostic tools for many medical and psychiatric disorders, including sleep disorders [1]. Deep learning and machine learning may have some advantages for disease classification compared to traditional mechanical classification. However, small or biased datasets can severely undermine accurate classification.

Digital therapeutics have also been introduced, including digital social rhythm therapy and digital cognitive behavioral therapy $[2,3]$. Telemedicine is also widely accessible, requiring only an internet connection; digital therapeutics and telemedicine can reduce both user time and cost. With digital therapeutics, clinicians can more easily check long-term compliance with behavioral guidelines for light exposure and sleep-time. Additionally, the use of digital therapeutics or telemedicine for contactless care can lower the risk of virus exposure in the current pandemic era. However, for most digital therapeutics, with the exception of digital therapy for insomnia, validation is still required; there are few

Received: September 11, 2021 Revised: September 14, 2021

Accepted: September 14, 2021

Corresponding author: Seog Ju Kim, MD, PhD, Department of Psychiatry, Sungkyunkwan University School of Medicine, Samsung Medical Center, 81 Irwon-ro, Gangnam-gu, Seoul 06351, Korea.

Tel: 82-2-3410-3589, Fax: 82-3410-0050, E-mail: ksj7126@skku.edu

(a) This is an Open Access article distributed under the terms of the Creative Commons Attribution Non-Commercial License (https://creativecommons.org/ licenses/by-nc/4.0) which permits unrestricted non-commercial use, distribution, and reproduction in any medium, provided the original work is properly cited. clinical guidelines or validation studies for auditory augmentation or guided relaxation (for sleep induction) using smartphones.

As consumer technologies, non-prescription devices directly marketed to consumers for circadian monitoring or intervention have also been introduced [4]. These include wearable devices, such as wristwatches, glasses, rings, and hairbands, as well as "nearable devices" that are placed near the bed. The smartwatch is a representative consumer technology. These devices can provide real-world, real-time information on sleep, activity, light exposure, and social rhythms. Some devices have sensors for the detection of more physiological circadian data, such as heart rate, temperature, or brain waves. Such devices are usually non-invasive and comfortable for consumers. Consumer sleep-tracking devices perform as well as research-grade actigraphs [5]; however, the safety and effectiveness of these devices for medical use is yet to be validated in most cases.

These new technologies can and should be integrated. Anonymized "big circadian data" obtained by wearable devices and smartphones would enhance the performance of machine learning in terms of detecting circadian problems. Wearable and nearable devices can promote long-term compliance with digital social rhythm therapy. Physiological data, which are obtained by wearable sensors and analyzed by artificial intelligence, may aid assessment of the effectiveness of digital therapeutics.

Along with these new technologies, digital phenotypes will become another form of non-verbal expression for patients, and classification of medical problems by machine learning will become an important tool for clinical diagnosis. Digital therapy should reduce the user burden. The current pandemic has accelerated these trends; many barriers to the use of these technologies in the medical field have recently been overcome. More flexible application of regulatory policy in this regard would help both clinicians and patients.

Although the new technologies discussed above are regarded as relatively harmless, concerns over the use of these new tools 
still remain. The low sensitivity and specificity of artificial intelligence and consumer devices for many diseases has yet to be resolved. Furthermore, access to these novel treatments does not guarantee enhanced compliance or efficacy. Lastly, new technologies may create another iatrogenic problem, just as the advent of electricity created the circadian rhythm disorder now known as shift work disorder. For example, "orthosomnia", i.e., preoccupation or concern with improving or perfecting sleep data, has emerged as a complication of wearable sleep tracking devices [6]. Over-enthusiasm, over-promise, or over-interest in these new technologies may lead to new complications. To overcome this, more validation and confirmation studies for these technologies are essential.

\section{Acknowledgments}

This work was supported by the Technology Innovation Infrastructure program through the Korea Institute for Advancement of Technology by The Ministry of Trade, Industry and Energy [No. P0014279], and a grant of the Korea Health Technology R\&D Project through the Korea Health Industry Development Institute (KHIDI), funded by the Ministry of Health \& Welfare, Republic of Korea (No. HR21C0885).

\section{Conflicts of Interest}

The author has no potential conflicts of interest to disclose.

ORCID iD

Seog Ju Kim (1D)

https://orcid.org/0000-0003-2467-5451

\section{REFERENCES}

1. Watson NF, Fernandez CR. Artificial intelligence and sleep: advancing sleep medicine. Sleep Med Rev 2021;59:101512.

2. Luik AI, van der Zweerde T, van Straten A, Lancee J. Digital delivery of cognitive behavioral therapy for insomnia. Curr Psychiatry Rep 2019;21:50.

3. Matthews M, Abdullah S, Murnane E, Voida S, Choudhury T, Gay G, et al. Development and evaluation of a smartphone-based measure of social rhythms for bipolar disorder. Assessment 2016;23:472-483.

4. Goldstein C. Current and future roles of consumer sleep technologies in sleep medicine. Sleep Med Clin 2020;15:391-408.

5. Depner CM, Cheng PC, Devine JK, Khosla S, de Zambotti M, Robillard R, et al. Wearable technologies for developing sleep and circadian biomarkers: a summary of workshop discussions. Sleep 2020;43:zsz254.

6. Baron KG, Abbott S, Jao N, Manalo N, Mullen R. Orthosomnia: are some patients taking the quantified self too far? J Clin Sleep Med 2017;13:351-354. 